\title{
Implicaciones políticas en la relación económica entre China y Estados Unidos, 1989-2000
}

$\mathrm{E}$ l objetivo central en este artículo es establecer el papel que han tenido Estados Unidos y la República Popular China en el desarrollo de la relación económica bilateral.

La actual interacción entre los dos países parte del supuesto de que la política entre ambas naciones ha influido fuertemente en la relación económica y comercial, especialmente tras el fin de la guerra fría y en el período del presidente norteamericano Bill Clinton, en cuya administración se plasmó un incesante crecimiento en el comercio entre ambas naciones. No obstante, es necesario especificar que lo económico también ha sido relevante en el desarrollo de la relación política entre ambos países. Con lo anterior se explica que la influencia entre los dos aspectos ha sido mutua.

Los objetivos esenciales de la interacción político-económica de China y Estados Unidos se fundamentan en lo siguiente. China tiene en cuenta que la relación política influye directamente en la interacción económica debido a que busca un desarrollo rápido y eficaz para su pueblo, que le permita convertirse en una potencia y que le ayude a legitimar el régimen actual. En ese sentido, Pekín ha ofrecido el peso de su convocatoria política en Asia del este no sólo para mostrar la trascendencia de su influencia en la región, sino también porque sabe que el apoyo a un ambiente de estabilidad regional es de suma importancia para alcanzar la meta de su propio desarrollo.

Para Estados Unidos la trascendencia de mantener una buena relación económica con China se enfoca en tres puntos esenciales. Pri-

* Investigador del Departamento de Estudios Internacionales, de la Universidad de Guadalajara. mero, Washington desea generar un mayor interés por parte de Pekín en los organismos internacionales de carácter político y económico. Segundo, Estados Unidos tiene presente que mediante el intercambio comercial y económico, China puede fortalecer su posición interna al lograr el desarrollo que persigue y reflejarlo al exterior; ello significa que una China estable representa una mayor seguridad regional acorde al propio interés estadounidense. Tercero, Washington desea que a través de su apoyo en el crecimiento económico de China, los líderes chinos se interesen en tomar una dirección de apertura democrática, y que ello, a su vez, se exprese en una interacción bilateral con menos fricciones.

De este modo, se puede establecer que la relación política entre Estados Unidos y la República Popular China presenta una dinámica de asociación, ya que los líderes chinos mantienen la idea de que el actual desarrollo económico de su país depende del mantenimiento de una buena relación política con los norteamericanos, mientras que para los estadounidenses una buena interacción económica con Pekín constituye un elemento esencial para lograr metas de cooperación en la relación política con China.

Las implicaciones políticas en la relación económica sino-estadounidense antes de 1989

Después del triunfo de la revolución comunista en 1949, y especialmente tras el rompimiento ideológico y político con la Unión Soviética, China optó por cerrar sus puertas al mundo. A finales de los setenta se implantó el modelo de puertas abiertas, el cual resultó en el rompimiento con la antigua política de cierre al comercio exterior que Mao Tse 
Tung había impuesto durante su mandato. Tras su muerte y acorde con una novedosa valoración de las relaciones económicas para el desarrollo de la nación, el nuevo líder, Deng Xiaoping, introdujo una serie de reformas dirigidas a buscar la modernización de su país por medio del intercambio comercial y la atracción de inversiones, valorando la relevancia de la interacción económica en la interacción mundial.

La apertura china se realizó bajo la consigna de que el propio régimen no podría sobrevivir si no acataba la realidad presente en cuanto a la trascendencia del comercio y a la necesidad de la inserción del Estado chino en el sistema del comercio mundial, como medio para alcanzar la modernización deseada. Así, la apertura económica, pero esencialmente el acercamiento político con Washington, benefició su vinculación con Estados Unidos y también comenzó a desarrollar de gran forma el intercambio comercial con sus vecinos de Asia del este.

Estados Unidos atrajo el interés económico de China a partir de una aproximación política, con el objetivo de establecer un proceso dirigido a disminuir las tensiones con $\mathrm{Pe}$ kín y ganarse su buena voluntad en la lucha por contener el expansionismo de la Unión Soviética en Asia del este.

Las autoridades norteamericanas ya habían concebido la idea de expandir su comercio con China desde la época de su acercamiento durante la administración de Richard Nixon. De hecho, desde 1971 Washington había levantado el embargo comercial impuesto contra China (González, 2000: 55) y durante 1973, en su visita a Pekín, el entonces secretario de Estado, Henry Kissinger comentó a su contraparte china (Chou Enlai) que
Pekín debería obtener los beneficios del tratamiento de Nación Más Favorecida (NMF). Sin embargo, esta situación no se presentó sino hasta la administración de Jimmy Carter (1976-1980).

En un principio la postura de Carter se había centrado en mantener una relación política balanceada con China, a través de la aplicación del principio de NMF. ${ }^{1}$ Era una forma de incentivar la apertura económica y también de presionar a Pekín para que accediera a una apertura política. De esta manera, el tratamiento de NMF se convirtió en una cuestión clave y de gran relieve simbólico en la relación política entre Estados Unidos y China (Mann, 1998: 107).

Con el objetivo de presionar dicha apertura, Washington amenazó con retirar los privilegios de NMF a China, basado en el acuerdo Jakson-Vanik. Esta ley, aprobada en 1974, planteaba que los beneficios del tratamiento de NMF de un país fueran retenidos si éste fallaba en permitir la libertad de emigración a sus ciudadanos. Y aunque al principio la recomendación estaba dirigida a la Unión Soviética (que no permitía la salida de sus ciudadanos judíos), la iniciativa se extendió en cierto sentido a China, que tampoco permitía que sus ciudadanos viajaran al extranjero (ibid.: 107).

Tras algunas desavenencias, en el verano de 1979 Estados Unidos otorgó el status de NMF a China. Asimismo, Carter decidió extender a Pekín financiamientos del Banco de Exportación-Importación y facilidades en los controles de exportación. De esta forma, sin mucho debate, el Congreso norteamericano autorizó el tratamiento de NMF para China, con el incentivo de ser renovado cada tres años (ibid.: 106). Asimismo, acordó que la renovación de dicho status estaría basado en la exis- 
tencia de un saldo comercial satisfactorio, así como en las condiciones y concesiones que el Congreso estadounidense estableciera (González, 2000: 54). No obstante, esta negociación se convirtió en la vía de acceso del comercio chino con el exterior y resultó benéfico para la inserción de China en la dinámica económica actual.

El cuadro 1 muestra la dinámica de intercambio comercial entre Estados Unidos y la República Popular China, desde 1978 hasta 1993.

\section{Las implicaciones políticas en la relación económica sino-estadounidense después de 1989}

Si las relaciones entre China y Estados Unidos habían sido significativamente constructivas en la época de su acercamiento entre 1972 y 1989, el colapso de la amenaza soviética transformó la estrategia política que Estados Unidos había estado desarrollando hacia China; de modo que las problemáticas en el campo económico y en el aspecto político se volvieron a hacer presentes. Además, la represión en Tiananmen en 1989 contribuyó para que se viera severamente afectada la perspectiva que Washington había tenido sobre una posible apertura política de China (Roy, 1998: 137).

De esta forma, tras los acontecimientos en Tiananmen, se presentaron embargos y sanciones por parte de Estados Unidos y sus aliados occidentales. La élite china se dio cuenta que la situación generada a partir de la repre- sión de los estudiantes, había provocado cierta incertidumbre en Washington, en el sentido de si China era una potencia aliada o enemiga (Roy, 1998: 137).

Los líderes chinos interpretaron las expresiones de Estados Unidos, como si China se tratara de un estado irresponsable, que no tenía consideración de la enorme contaminación ambiental que estaba produciendo en el curso de su desarrollo económico con pruebas nucleares, y que además generaba cierta incertidumbre por la venta de armas -dirigida principalmente a países musulmanes- en el mercado mundial, actos, que otras grandes potencias ya habían hecho anteriormente. Además, algunos líderes chinos percibieron la actitud de Washington como un intento para forzar a Pekín a acatar una serie de reglas estadounidenses que no estaban dispuestos a seguir. Posteriormente, aunque las tensiones entre los dos países disminuyeron con motivo de la visita a China de una delegación norteamericana, encabezada por el encargado para la seguridad nacional, Brent Scowcroft, asimismo por el hecho de continuar recibiendo trato igualitario en el comercio internacional basado en el principio de NMF, la desconfianza en Pekín permaneció (Mann, 1998: 181).

Aprovechando su enorme influencia, Washington desplegó una dinámica para castigar significativamente a China. Esta situación se expresó incluso en el fracaso de Pekín durante la votación a mediados de los noventa para obtener la sede de los juegos olímpicos de 2000, así como en el bloqueo norteamericano para impedir que China entrara en la Or-

\begin{tabular}{cccc}
\multicolumn{5}{c}{$\begin{array}{c}\text { Cuadro 1 } \\
\text { Intercambio comercial sino-estadounidense 1978-1993 }\end{array}$} \\
\hline Año & $\begin{array}{c}\text { Exportaciones de EU a } \\
\text { China (millones de dólares) }\end{array}$ & $\begin{array}{c}\text { Importaciones de EU } \\
\text { provenientes de China } \\
\text { (millones de dólares) }\end{array}$ & $\begin{array}{c}\text { Balanza } \\
\text { comercial }\end{array}$ \\
\hline 1978 & 821 & 324 & 497 \\
1982 & 2,912 & 2,284 & 628 \\
1983 & 2,173 & 2,244 & -68 \\
1992 & 7,418 & 25,728 & $-18,309$ \\
1993 & 6,318 & 23,013 & $-16,695$ \\
\hline
\end{tabular}

Fuente: US Department of Commerce. 
ganización Mundial de Comercio desde un principio (Roy, 1998: 136).

Por su parte, a pesar del deseo de Pekín de diversificar su mercado para disminuir una potencial vulnerabilidad, Estados Unidos se había convertido en el principal destino de sus exportaciones y en uno de sus principales inversores. Del mismo modo, su comercio bilateral se estaba incrementando en un promedio de $25 \%$ anual a principios de la década de los noventa. Sin embargo, las mismas disputas políticas entre las dos naciones no habían evitado que China se viera beneficiada por los flujos de capital provenientes de Norteamérica (Roy, 1998: 138). Esta situación es mostrada en el cuadro 2.

Cuando Bill Clinton comenzó su administración en 1993, se aparentaba que China no estaba en la lista de prioridades para el gobierno norteamericano. No obstante, Warren Christopher, el nuevo secretario de Estado, comentó que la política estadounidense pretendía que en China se diera una transición pacífica del comunismo a la democracia (Mann, 1998: 277).

La administración Clinton se centró en presionar para que mejoraran los derechos humanos al interior de China. Comenzó a ponerse en duda el continuar otorgando a China la premisa de $\mathrm{NMF}$, argumentando la constante violación a los derechos humanos por parte del gobierno chino. De hecho, Clinton apoyó la idea de revocar el tratamiento de NMF en todos los productos chinos, si Pekín no aplicaba los principios mínimos establecidos sobre las normas de derechos humanos, comercio internacional y proliferación de armas (Lilley y Wilkie, 1995: 204). No obstante, aunque en un momento se pensó tratar por separado los problemas de comercio y control de armamento, del tratamiento de $\mathrm{NMF}$, la aparición de los primeros saldos negativos en la balanza comercial de Estados Unidos con China, también influyeron de forma determinante en la posibilidad de seguir otorgando a China o no, el principio de NMF (González, 2000: 54).

Sin embargo, tras los problemas presentados por la actitud de Corea del Norte, se concibió que la participación china en la problemática era de gran importancia para la estabilidad de la península coreana, de modo que la negociación de dicho status volvió a favorecer a Pekín. El triunfo fue de mayor relevancia debido a que en 1994 Clinton anunció que Estados Unidos no debería condicionar más a China en los beneficios del tratamiento de NMF, a costa de las mejoras que presentara Pekín en materia de derechos humanos (Roy, 1998: 138).

Clinton también pensó que un acuerdo político y económico podría ayudar a que Pekín desarrollara una conducta de cooperación (Lilley y Wilkie, 1995: 202). Durante esa época la balanza comercial norteamericana, que siempre había salido bien librada, comenzó a presentar problemas en 1994, cuando el intercambio empezó a favorecer a China. Esta situación queda ilustrada en el cuadro 3.

Asimismo, la relación con China comenzó a verse con más interés en el campo económico por parte de los inversionistas norteamericanos, debido a su trascendencia

Cuadro 2

Inversiones en China provenientes de Estados Unidos

\begin{tabular}{cc}
\hline Año & $\begin{array}{c}\text { Cantidad de inversión estadounidense } \\
\text { (millones de dólares) }\end{array}$ \\
\hline 1990 & 3,487 \\
1993 & 27,515 \\
1998 & 45,463 \\
1999 & 40,715 \\
2000 & 40,842 \\
\hline
\end{tabular}

Fuentes: MOFTEC, The United States-China Business Council.

Ministry of Foreing Trade and Economic Cooperation. 
como fuente de mano de obra barata, así como un potencial mercado de 1300 millones de habitantes (con capacidad adquisitiva para consumir productos norteamericanos).

Entre 1995 y 1996, las relaciones sinonorteamericanas se vieron nuevamente afectadas debido a las maniobras militares del ejército chino frente a Taiwan en la víspera de la celebración de elecciones internas de la isla. Sin embargo, a partir de 1997 se renovó el título de NMF acabando con las restricciones por cuestiones laborales de forma definitiva y se terminó con la incertidumbre de cada tres años (González, 2000: 54). Posteriormente, en junio de 1998, el presidente Clinton realizó un viaje a China para firmar acuerdos sobre exportación de alta tecnología, desarrollo de infraestructura y aplicaciones de Internet (González, 2002: 15). Tras su regreso a Washington reinició sus planes para obtener la aprobación del Congreso para la entrada de China a la Organización Mundial del Comercio y renovar las relaciones comerciales normales con Pekín.

Poco antes de la reunión ministerial de 1999 en Seattle, Estados Unidos y China concluyeron un acuerdo que involucraba concesiones importantes para China, otorgando a las compañías extranjeras acceso en los servicios financieros y en otros sectores sensibles, bajo la premisa de liberar el sector de las industrias pesadas manufactureras pertenecientes al estado. A su vez, permitiría a Washington el uso de programas especiales para proteger sus industrias contra los enor- mes flujos de importaciones. Finalmente, en noviembre de 1999, Estados Unidos y China firmaron un acuerdo sobre los términos de acceso a los que Pekín quedaba expuesto para su ingreso a la Organización Mundial de Comercio (González, 2002: 15).

De esta forma, a pesar de que tras el fin de la guerra fría la relación política entre China y Estados Unidos se vio un tanto afectada y que trascendió de alguna forma en lo económico, el intercambio comercial y la inversión estadounidense en China, lejos de disminuir se incrementó a grado tal que sentó las bases para que China pudiera entrar a la omc.

\section{La identidad y la legitimidad en la relación económica sino-estadounidense}

Las implicaciones políticas en la relación económica sino-estadounidense plantean la existencia de elementos que se interrelacionan, creando una interacción de ida y vuelta donde la necesidad de una buena relación política incentiva la necesidad de enlaces de otros tipos en los cuales el intercambio económico es de suma importancia. Al mismo tiempo, el intercambio económico puede servir a ciertos propósitos que un estado puede tener en relación al otro.

Después de los rezagos y calamidades sociales, políticas y económicas que trajeron el Gran Salto Adelante y la Revolución Cultural, y aún después de la muerte de Mao Tse Tung, la dirigencia china se trabó en una lu-

Cuadro 3

Intercambio comercial sino-estadounidense, 1994-2000 (millones de dólares)

\begin{tabular}{cccc}
\hline Año & $\begin{array}{c}\text { Exportaciones de EU } \\
\text { a China }\end{array}$ & $\begin{array}{c}\text { Importaciones } \\
\text { provenientes de China }\end{array}$ & Balanza comercial \\
\hline 1994 & 9,300 & 41,400 & $-35,700$ \\
1995 & 11,800 & 48,500 & $-36,700$ \\
1996 & 12,000 & 54,400 & $-42,200$ \\
1997 & 12,800 & 65,800 & $-53,000$ \\
1998 & 14,300 & 75,100 & $-60,800$ \\
1999 & 13,100 & 109,400 & $-74,700$ \\
2000 & 16,300 & 107,600 & $-91,200$ \\
\hline
\end{tabular}

Fuentes: US International Trade Comission, US Department of Commerce.

The United States-China Business Council. 
cha que derivó en el ascenso al poder de uno de los llamados liberales, Deng Xiaoping.

Consciente del severo atraso económico que sufría el país, Deng vislumbró que China necesitaba una transformación profunda que le permitiese alcanzar la modernidad y el desarrollo económico a la par de las grandes potencias. Asimismo, se proyectó a recuperar la legitimidad política del Partido Comunista Chino (PCC), tan fuertemente dañada por los acontecimientos anteriores.

Deng dio prioridad a la necesidad de alcanzar la modernización de la industria, la agricultura, la defensa nacional, la ciencia y la tecnología. Con ese objetivo permitió que el campo político quedara supeditado a la necesidad económica. Asimismo, si durante la era de Mao la valoración de los dirigentes chinos sobre el imperialismo, fuese norteamericano o soviético, dirigiría inevitablemente a una confrontación, en la era de Deng los dirigentes chinos comenzaron a descartar esa situación. De esta forma, China decidió disminuir al máximo su apoyo a estados revolucionarios y a los movimientos nacionalistas, y se dispuso a centrar todas sus fuerzas en su propio desarrollo económico; abandonó su aislamiento y comenzó una política de apertura que resultó en un incremento constante de intercambios con el exterior, principalmente con Estados Unidos. A su vez, esto contribuyó a ubicar a China en la realidad mundial en la lucha por modernizarse (Chen, 2000: 414).

Estados Unidos, que en un principio sólo vislumbró la importancia geoestratégica de China en la contención de la Unión Soviética en Asia del este, observó que la inserción de Pekín en la economía mundial permitiría cumplir con una serie de objetivos fijados en el rubro político y económico, es decir, que se presentara la posibilidad de que una apertura económica incidiera en una posible apertura política al interior del país y que ello se tradujera en un proceso de democratización y de avance en la cuestión de los derechos humanos.
Sin embargo, también abarca el campo de los beneficios económicos mutuos (Conable y Lampton, 1993: 134). Estados Unidos está consciente del enorme potencial del mercado interno chino, de su mano de obra barata y de la necesidad china de obtener tecnología de primer nivel, de modo que el establecimiento de un intercambio económico a cambio de una cooperación política beneficiosa no representa realmente un gran sacrificio para Washington, sino que aporta una positiva dinámica económica de la cual también obtiene beneficios.

En referencia a lo anterior, Estados Unidos también tuvo presente que las medidas para procurarse una relación benéfica y cooperativa requerían de un constante mantenimiento del ambiente de paz y estabilidad (Kirshner, 1998: 75). En ese sentido, si Washington hubiera tomado la determinación de disminuir sus contactos comerciales u obstruir la entrada de Pekín en los organismos internacionales de comercio, la posibilidad de lograr avances al interior de China en la promoción de valores como la democracia, el respeto a los derechos humanos y el acatamiento de los principios de los organismos internacionales promovidos por Washington, probablemente se hubiera visto afectada.

Asimismo, Estados Unidos ha tenido que tomar en cuenta los deseos de China de ampliar el número de sus enlaces económicos y de ser aceptada por los organismos internacionales. De este modo, ha aprovechado su poder económico en dichos organismos para interesar a Pekín en procurar el acatamiento de las reglas que éstos imponen. Al mismo tiempo, mantiene cierto control sobre algún intento chino de afectar el orden del sistema internacional.

Por su parte, para China la necesidad de crecimiento económico no ha implicado que haya tenido que supeditarse del todo al acatamiento de las reglas impuestas por la influencia del poder económico de Estados Unidos, a pesar de algunas eventualidades como la revocación del tratamiento de NMF tras los acontecimientos de Tiananmen en 1989. De hecho, 
en 1997 Washington eliminó las restricciones de carácter laboral y dio el primer paso a la negociación de la entrada de China a la omc. Así, se puede decir que fue Estados Unidos el que tuvo que supeditarse a la realidad que representa China y no a una dinámica deseada, asumiendo con ello que una buena relación económica con China se traduce en la promoción de una relación política estable y en la estabilidad regional de Asia.

\section{Importancia del poder y seguridad nacional}

Todos los estados se ven obligados por la realidad a procurarse la obtención de capacidad material de recursos. No obstante, el poder se constituye en cuanto al significado que le otorga el interés (Wendt, 1999: 109). Con ello se sostiene que la motivación de un actor (Estado), determinada por una serie de valores permite mostrar una señal intersubjetiva, pero más completa de su poder.

La relación política sino-norteamericana incide en su relación económica debido a que ambas se ven influenciadas por la realidad que han construido. En la actualidad el intercambio comercial entre las dos potencias se ha incrementado de manera notable, y de la misma forma que el mercado norteamericano es indispensable en el crecimiento económico de China, al menos por el momento, para Estados Unidos la mano de obra barata china, así como su potencial mercado interno de más de 1300 millones de personas motivan a la continuidad de una buena relación económica.

En su lucha por continuar con el fortalecimiento y la supervivencia de un sistema político y social, los líderes chinos buscan seguir con el ascenso económico de su país, y para ello tienen presente que una buena relación económica con Estados Unidos no sólo incide en su desarrollo (porque tiene el mercado más importante del mundo o la tecnología de punta más avanzada en varios sectores), sino también porque cuenta con potenciales fuentes de crédito e inversiones (Anguiano, 1997: 46), a través de las cuales el régimen de Pekín se ha visto fuertemente beneficiado, debido a que se ha vuelto uno de sus mayores receptores.

En lo referente al comercio exterior, la necesidad de China de lograr su admisión en la Organización Mundial de Comercio se enfoca en las oportunidades y las ventajas que la organización ofrece a sus miembros, como el fortalecimiento del prestigio como un poder estable y responsable; la posibilidad de incrementar su comercio debido a la reducción de las amenazas de proteccionismo contra las exportaciones chinas; la provisión del régimen de servicios de valor económico como reglas y expectativas; información de mercado y procedimientos para resolver disputas.

Asimismo, se presenta la dificultad para Estados Unidos de aplicar sanciones económicas para castigar a China por cuestiones políticas, como el respeto a los derechos humanos, aunque Washington sigue contando con la enmienda Jackson-Vanik, la cual precede cualquier acuerdo comercial al que Estados Unidos desee entrar (Roy, 1998: 94).

Washington ha tratado de utilizar su poder económico tanto en el comercio como en los organismos internacionales, con el objetivo de ejercer una mayor influencia sobre las decisiones políticas que toma el gobierno chino. Estados Unidos ha expresado su poder, difundido por varias vías indirectas de la estructura económica internacional. En ese sentido, el poder de Estados Unidos es capaz de influir fuertemente en el funcionamiento de la política económica global. No obstante, también habría que señalar que dicho poder no se centra totalmente en Estados Unidos; esto se debe a que Washington también ha sido influido por los atractivos económicos y comerciales que China ofrece, los cuales han desencadenado un constante déficit comercial con el gigante asiático.

Sin embargo, el punto esencial se centra sobre la presente realidad de que Washington necesita el poder diplomático regional de China para mantener cierto apoyo en una cuestión considerada como de seguridad nacional, 
la estabilidad en Asia del este, más concretamente en la península de Corea. Bajo ese contexto, China no sólo ha superado en muchas ocasiones las políticas restrictivas norteamericanas como la enmienda Jackson-Vanik, sino que también, en reciprocidad por su apoyo en la cuestión de Corea, recibió su recompensa con la firma del acuerdo bilateral en 1997 (Mann, 1998: 332).

Lo dicho en el párrafo anterior indica que la relación económica también es influenciada por las cuestiones de seguridad nacional. Dicha influencia no es ligera ni estrecha, sino de gran relevancia, y es en ese rubro en donde las implicaciones políticas exhiben con más claridad la fuerza de su incidencia sobre la relación económica. Así, se presenta una dinámica en la que Estados Unidos cuenta con la capacidad de influir en cuestiones económicas mientras que China cuenta con una importante dimensión mediadora para la estabilidad política de Asia del este.

Según Peter Katzenstein, la seguridad nacional no sólo se limita al aspecto militar, sino también al efecto que los acontecimientos tanto externos como internos tienen sobre la calidad de vida de la población de un país (Katzenstein, 1996: 8). En ese sentido, Washington pugna por promover el interés chino en cuestiones de seguridad nacional en territorio de Asia del este, con el fin de continuar con un ambiente relativamente estable, lo cual le ha beneficiado. A su vez, Pekín trata de mostrar a Estados Unidos que es importante que prosiga con una buena relación económica a fin de permitir que su crecimiento económico continúe, lo cual propicia una situación esencial, no sólo para mantener la seguridad nacional china, sino también la estabilidad regional.

Para Estados Unidos la relevancia de entablar una relación económica con China también evoca el interés de promover cambios democráticos al interior del régimen chino. Los líderes norteamericanos tienen la esperanza de que la instrumentación de reformas económicas y su propia interacción proporcione ba- ses para el crecimiento de la economía privada y otorgue una mayor participación a la sociedad civil china, y que ello a su vez se traduzca en la producción de una política democrática liberal que disminuya la "amenaza" china (Lieberthal, 1995: 37).

En su momento, el presidente Bill Clinton manifestó que un acuerdo político y económico con una de las economías globales de mayor crecimiento no sólo debería garantizar el apoyo de un comportamiento cooperativo de Pekín, sino también una dinámica de promoción que podría guiar a una evolución pacífica hacia la democracia (Lilley y Wilkie, 1995: 202).

\section{Conclusión}

Según Barber Conable y David Lampton, la política económica estadounidense desarrollada por la administración Clinton hacia China ha estado encaminada a propiciar de manera gradual, la creación de mejores condiciones para una sociedad más plural y un gobierno más abierto. A través de una amplia participación en la economía de China, Estados Unidos interesó a Pekín en la liberalización económico-comercial de sus ciudadanos, así como en el cuidado de los derechos humanos.

Sin embargo, para China la necesidad de crecimiento económico no implicó que tuviera que supeditarse del todo al acatamiento de las reglas impuestas por la influencia del poder económico de Estados Unidos. Incluso, en palabras de Eugenio Anguiano, algunos líderes chinos mantienen, hasta la fecha, la idea de que dadas las características de su gobierno, así como del tamaño de su población, lo más conveniente para la estabilidad interna y externa es una China rica, políticamente monolítica y con una economía controlada desde el centro, y no una China liberalizada, descentralizada y con una economía supeditada al juego liberal de la oferta y la demanda que se traduzca en un desarrollo desigual interno que motive movimientos de fuerzas centrífugas, o que permita una descontrolada emigración de millones de trabajadores chinos a los países 
más industrializados, posibilitando con ello la presentación de un ambiente de desestabilización que afectaría no solamente a China, sino también a otros países.

Estados Unidos tiene la necesidad de comprometerse con la adopción de una posición que mueva la relación política y económica con China en una dirección menos emocional, y debe tener presente que el mantener cierta estabilidad regional es una cuestión prioritaria que está por encima de la promoción de elementos como los derechos humanos y los valores democráticos.

A final de cuentas, para mantener la estabilidad regional y no afectar su propia seguridad nacional o la de sus socios del este asiático, Washington necesita una buena relación económica y política con China, porque si decide contener el crecimiento económico chino, proporcionará elementos suficientes para que las fuerzas nacionalistas y militaristas refuercen sus posiciones al interior de China, creando un ambiente de antagonismo político.

Lo anterior significa que existe el peligro de que dichas fuerzas proyecten con mayor vehemencia una posición que afecte no sólo la estabilidad en Asia del este, sino fundamentalmente, la relación política con Estados Unidos. Con ello se reforzaría la posibilidad de presentarse un ambiente antagónico entre las dos naciones.

\section{Bibliografía}

Anguiano, Eugenio (2001) “China contemporánea: la construcción de un país (desde 1949)". Programa de estudios APEC. Centro de Estudios de Asia y África. El Colegio de México.

Anguiano, Eugenio (2000) "Seguridad regional Asia Pacífico”. Centro de Estudios del Pacífico. El Colegio de México.

Anguiano, Eugenio (1997) "Seguridad Regional en Asia Pacífico". Centro de Estudios del Pacífico. El Colegio de México, pp. 27-67.

Chan, Steve (1992) "National Security in the Asia-Pacific: Linkages Among Growth, Democracy and Peace". Contemporary Southeast Asia. Institute of Southeast Asian Studies, vol. 14, núm.1, pp. 13-32.
Chen, Jian (2000) China en transición: Sociedad, cultura y economía. En Taciana Fisac y Steve Tsang editores. Editorial Bellaterra. Barcelona, España, pp. 393-419.

Christensen, Thomas J. (2001) "Posing Problems without Catching Up: China's Rise and Challenges for U.S. Security Policy”. International Security, vol. 25, núm. 4 (primavera), pp. 5-40.

Christensen, Thomas J. and Betts, Richard K. (2000-2001). "China: Getting the questions right". The National Interest. Washington, United States, núm. 62 (primavera), pp. 1729.

Collins, Alan. (2000) The Security Dilemas of Southeast Asia. MacMillan Press LTD. London, England.

Conable, Barber B. y David M. Lampton (1992-1993) "China: The Coming Power”. Foreing Affairs, pp. 133-149.

Cornejo, Romer A. (1998). "China: la consolidación de un nuevo liderazgo y una exitosa democracia". Asia-Pacífico. Centro de Estudios del Pacífico. El Colegio de México, pp. 111-123.

Faigenbaum, Evan A. (2001). "China's Challenge to Pax Americana”. The Washington Quarterly. The Center for Strategic and International Studies and the Massachusetts Institute of Technology. Vol. 24, núm. 3, pp. 31-43.

Gilboy, George y Heginbotham, Eric (2001) "China: la transformación que viene”, Foreing Affairs. Vol. 80, núm. 4, pp. 237-252.

Gilpin, Robert (1987) The Political Economy of International Relations, Princeton, Princeton University, USA.

González, Juan (2002) "La política comercial de la RP China y su aceptación-regreso a la OMC". Estudios del Pacífico. El Colegio de México, pp. 1-30.

González, Juan (2000) "Estados Unidos: Acuerdo bilateral con China para su acceso a la Organización Mundial de Comercio". México y la Cuenca del Pacífico. Departamento de Estudios del Pacífico. Universidad de Guadalajara. Enero-abril. vol. 3, pp. 53-60.

Hopf, Ted (1998) "The Promise of Constructuvism in International Relations Theory". International Security, vol. 23, pp. 172-200.

Horowitz, Shale (2001) "Reconstructing Conflict in East Asia: Preserving Two Koreas, and Moving towards One China and One Taiwan. Pacific Focus. Center of International Studies, Inha University. Vol. XVI, núm. 1 (primavera), pp. 35-55.

Hurd, Ian (1999) "Legitimacy and Authority in International Politics". International Organizations, vol. 53, núm. 2 (primavera), pp. 379-408. 
Johnston, Alastair I. (1996) Cultural Realism and Strategy in Maoist China. En Katzesntein Peter. Columbia University Press. New York, pp. 217-268.

Katzestein, Peter (1996) The Culture of National Security: Norms and Identity World Politics. Columbia University Press. New York.

Kim, Samuel S. (1998) “China on the World: chinese foreing policy faces the new millennium”. Westview Press.

Levine, I Steven (1998) "Sino-american relations: Practicing Damage Control”. En Kim Samuel S. Westview Press, pp. 91-113.

Lieberthal, Kenneth (1995) "A New China Strategy". Foreing Affairs. Vol. 74, núm. 6, pp. 35-49.

Lilley, James (1994) "Freedom through trade". Foreing Policy, núm. 94 (primavera), pp. 37-43.

Lilley, James and Wilkie, Wendell L. (1995) "Beyond MFN: Trade with China and American Interest". The American Asian Review. Washington D.C. vol. XIII, núm. 1 (primavera), pp. 202-210.

Mann, James (1998) A history of Americas curious relationships with China, from Nixon to Clinton. Vintage Book.

Roy, Denny (1998). China's Foreing Policy. Macmillan Press LTD. England.

(1998) "Current Sino-U.S. relations in strategic perspective”. Contemporary Southeast Asia. Vol. 20, núm. 3, Singapur, pp. 225-240.

(1994) "Hegemon on the Horizon?: China's Threat to East Asian Security". International Security. Vol. 19, núm. 1, pp. 149-168.

(1997) "The Foreing Policy of Great-Power China”. Contemporary Southeast Asia. Institute of Southeast Asian Studies. vol. 19, núm. 2 (septiembre), pp. 121-135.

Segal, Gerald (1999) “Does Chinas Matter?”. Foreing Affairs. vol. 78, núm. 5, pp. 24-36.

Shambaugh, David (2001) "Facing reality in China Policy". Foreing Affairs. Jan/Feb. New York, United States. vol. 80, núm. 1, pp. 50-64.
Wendt, Alexander (1992) “Anarchy is what States Make of it: The Social Construction of Power Politics". International Organization, vol. 46, núm. 2 (primavera), pp. 391-425.

Wendt, Alexander (1999) Social Theory of International Politics. "Ideas all the way down?: on the constitution of power and interest". Cambridge University Press. United Kingdom.

Wilhelmy, Manfred (2000) "El proceso de reformas en China y la política exterior”. Estudios del Pacífico. El Colegio de México. Núm. 2, pp. 1-45.

Wu, Ximbo (2000) "U.S. Security Policy in Asia: Implications for China-U.S. Relations". Foreing Policy Studies. Center for Northeast Asian Policy Studies Shanghai China. (septiembre).

Yuan, Jing-Dong (1995) "China's Defence Modernization: Implications for Asia-Pacific Security”, Contemporary Southeast Asia. Institute of Southeast Asian Studies. vol. 17, núm.1.

\section{0 tras fuentes}

U.S.-China Policy Review Main Page (1998)

\section{Fuentes estadísticas}

US Departament of Commerce.

MOFTEC The United States-China Business Council.

Ministry of Foreing Trade and Economic Cooperation.

\section{Nota}

1 El principio de Nación Más Favorecida parece sugerir que se trata de algún tipo de trato especial para un país determinado, pero en la omc significa realmente la no discriminación. En virtud de los acuerdos de la omc, los países no pueden por regla general establecer discriminaciones entre sus diversos interlocutores comerciales. Si se concede a un país una ventaja especial (por ejemplo, la reducción del tipo arancelario aplicable a uno de sus productos), se tiene que hacer lo mismo con todos los demás miembros de la омc. Ty:- 